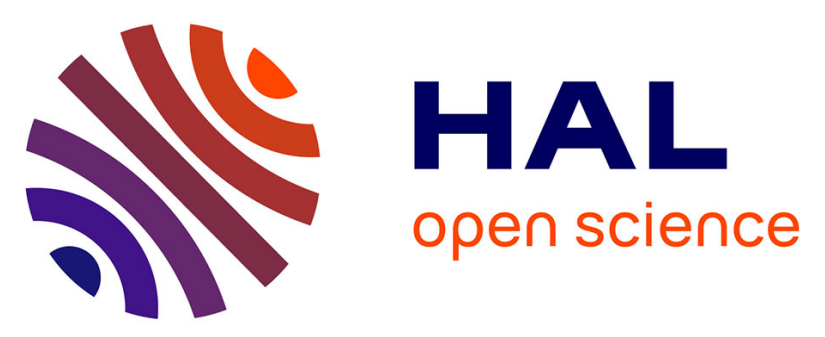

\title{
Evaluation of micro laser sintering metal 3D-printing technology for the development of waveguide passive devices up to $325 \mathrm{GHz}$
}

Victor Fiorese, Cybelle Belem-Gonçalves, Carlos del Rio, Diane Titz, Frédéric Gianesello, Cyril Luxey, Guillaume Ducournau, Emmanuel Dubois, Christophe Gaquière, Daniel Gloria

\section{To cite this version:}

Victor Fiorese, Cybelle Belem-Gonçalves, Carlos del Rio, Diane Titz, Frédéric Gianesello, et al.. Evaluation of micro laser sintering metal 3D-printing technology for the development of waveguide passive devices up to $325 \mathrm{GHz}$. IEEE/MTT-S International Microwave Symposium, IMS 2020, Aug 2020, Los Angeles, United States. pp.1168-1171, 10.1109/IMS30576.2020.9224102 . hal-03091222

\section{HAL Id: hal-03091222 \\ https://hal.science/hal-03091222}

Submitted on 5 Jan 2021

HAL is a multi-disciplinary open access archive for the deposit and dissemination of scientific research documents, whether they are published or not. The documents may come from teaching and research institutions in France or abroad, or from public or private research centers.
L'archive ouverte pluridisciplinaire HAL, est destinée au dépôt et à la diffusion de documents scientifiques de niveau recherche, publiés ou non, émanant des établissements d'enseignement et de recherche français ou étrangers, des laboratoires publics ou privés. 


\title{
Evaluation of Micro Laser Sintering Metal 3D-Printing Technology for the Development of Waveguide Passive Devices up to $325 \mathrm{GHz}$
}

\author{
V. Fiorese ${ }^{1,2}$, C. Belem Gonçalves ${ }^{1,2}$, C. del Rio Bocio ${ }^{3}$, D. Titz ${ }^{4}$, F. Gianesello ${ }^{1}$, C. Luxey ${ }^{4}$, \\ G. Ducournau ${ }^{2}$, E. Dubois ${ }^{2}$, C. Gaquière ${ }^{2} \&$ D. Gloria ${ }^{1}$ \\ ${ }^{1}$ STMicroelectronics, 850 rue Jean Monnet, 38920 Crolles, France \\ ${ }^{2}$ IEMN, UMR CNRS 8520, Avenue Poincaré-CS 60069, 59652 Villeneuve-d'Ascq, France \\ ${ }^{3}$ Electrical and Electronic Engineering Department, Public University of Navarre, Pamplona, 31006 Spain \\ ${ }^{4}$ Polytech'Lab Nice-Sophia, Univ. Nice Sophia-Antipolis, Sophia Antipolis, 06903 France
}

\begin{abstract}
In this paper, we propose an assessment up to 325 $\mathrm{GHz}$ of Micro Laser Sintering (MLS) metal 3D-Printing technology in order to achieve lightweight and cost-effective millimeter wave $(\mathrm{mmW})$ passive function. We first designed and manufactured a bended WR5 waveguide in order to assess achievable roughness and insertion loss. In a second step, an existing $240 \mathrm{GHz}$ choke horn antenna design, previously manufactured using metal coated Stereo Lithography Apparatus (SLA) and Selective Laser Melting (SLM) technologies, has been prototyped using MLS. Measured performances of the MLS antenna prototype have been benchmarked with SLA and DMLS ones. Achieved performances are promising since without any post processing MLS compete up to $325 \mathrm{GHz}$ with metal coated SLA technology while it enables a metallic part manufactured in a single piece.

Keywords $-\mathrm{mmW}$, THz, additive manufacturing, 3D-Printing, waveguide, horn antenna.
\end{abstract}

\section{INTRODUCTION}

The never-ending demand of consumers for more data access requires wireless technology to increase achievable data rate. The current deployment of $5 \mathrm{G}$ is a good example and looking more in the future the 200 and $320 \mathrm{GHz}$ frequency bands constitute an interesting window with preliminary IEEE802.15.3d standard being promoted [1].

III-V technologies have been for a long time the unique ones able to address $\mathrm{mmW}$ applications, but advanced silicon technologies capabilities enable today interesting potential in the $\mathrm{mmW}$ and the $\mathrm{THz}$ spectra from 0.1 to $1 \mathrm{THz}$ [2] paving the way for cost-effective solutions required by the mass market. Various CMOS and BiCMOS wireless $\mathrm{mmW}$ systems have already been demonstrated such as long-range radar in $\mathrm{W}$ band (75-110 GHz) [3], point to point wireless backhaul link (110$170 \mathrm{GHz})$ [4] next gen Radars in G band (140-220 GHz) [5].

However, the deployment on the consumer market using silicon-based mmW system operating beyond $200 \mathrm{GHz}$ will require the development of innovative and cost-effective packaging technology. Classically, the packaging of $\mathrm{mmW}$ and $\mathrm{THz}$ circuits is achieved using metal waveguide based assembly [6]. These packages are machined from metal utilizing computer numerically controlled (CNC) milling. While excellent performances can be achieved with such approach, its cost structure and complexity is not compatible with the consumer market. To address this challenge, micromachined waveguide packaging techniques (DRIE-based silicon micromachined packaging in particular) have emerged as a promising solution to develop a low loss and cost-effective packaging solution [7].

Recently, 3D-Printing technologies have been considered to complement micromachined waveguide packaging techniques and promising results have been reported up to $330 \mathrm{GHz}$ [8]. Contrary to micromachining, 3D-Printing is an additive manufacturing technique meaning that $3 \mathrm{D}$ printed devices are built up layer by layer following a tooling free process. This implies a shorter manufacturing time and cost savings due to both the absence of tooling costs and reduced wastes.

Historically, dielectric 3-D-printed devices, generally built in split pieces requiring electroplating and assembly, have been considered first. Impressive results (competing traditionally fabricated commercial counterparts) have been reported in $\mathrm{H}$ band (220-325 GHz) for waveguides and diagonal horn manufactured using SLA [9]. While being lightweight, metal coated dielectric 3D printed devices feature complex process (coating being challenging for complex shapes). Consequently, metallic 3-D-printed devices have emerged as an appealing solution for the development of waveguide based component.

SLM metallic 3D-Printing technology (using $\mathrm{Cu}-15 \mathrm{Sn}$ powder with $<20 \mu \mathrm{m}$ particle size) has already been evaluated up to H-band [10]. Surface roughness of SLM technology being a limiting factor, post treatments (manually polishing, goldelectroplating and micromachined process [11]) have been applied enabling to achieve $\mathrm{H}$-band horn antenna performances competing with traditional $\mathrm{CNC}$ machined part. However, achievable surface roughness $(\sim 6 \mu \mathrm{m})$ has limited printed waveguide performances using SLM [12] since post treatment using abrasive-particle-contained high-pressure fluid to wash through the waveguide (like micromachined process) exhibits limited efficiency for long and small aperture $(<3 \mathrm{~mm})$ (the fluid pressure decreasing dramatically along the waveguide). Consequently, the waveguides attenuation manufactured using SLM has been so far acceptable up to D-band.

To improve achievable performances up to H-band, we propose to evaluate MLS technology, which uses powder with particle sizes $<5 \mu \mathrm{m}$, to manufacture 3D printed metal parts with improved surface roughness (without any post processing) and consequently reduced loss. In section II we introduce the MLS technology, in section III achieved performances of MLS waveguide and horn antenna in H-band are reviewed and section IV provides some conclusion. 


\section{Micro LASER Sintering Metal 3D-Printing TECHNOLOGY OVERVIEW}

Three main technologies exist today for metallic 3Dprinting. The first one, Binder Jetting (BJ) is selectively depositing a liquid binding agent to join metal powder particles. The process of binder printing and powder recoating is repeated until completion. The final object is placed in a convection oven to dry all parts. The second technology is Selective Laser Sintering (SLS) which uses a laser as the power source to sinter powdered material according to the 3D model of targeted part. After each cross-section is scanned, the powder bed is lowered by one layer thickness, a new layer of material is applied on top, and the process is repeated until the part is completed. The last technology is SLM which is considered as a subcategory of SLS. It consists in selective melting and fusing of powder material using a high power-density laser beam. A liquid pool is formed and cooled down afterwards. The SLM process has the ability to fully melt the metal material into a solid three-dimensional part unlike SLS.

Each metallic 3D-Printing technology can be related to two key parameters: dimensional control and surface roughness. We propose in Table 1 a quick benchmark of achievable performances using BJ, SLS and SLM technologies:

Table 1. Tolerances of well-known 3D-Printing techniques

\begin{tabular}{|c|c|c|c|}
\hline & BJ & SLS & SLM \\
\hline Material & powder & powder & powder \\
\hline $\begin{array}{c}\text { Dimensional } \\
\text { control }\end{array}$ & medium & medium & tight \\
\hline $\begin{array}{c}\text { Surface } \\
\text { roughness }\end{array}$ & $4 \mu \mathrm{m}$ & $2 \mu \mathrm{m}$ & $2 \mu \mathrm{m}$ \\
\hline
\end{tabular}

For metallic 3D-Printing, SLS and SLM are the two best options. For example, SLS provides a typical $10 \mu \mathrm{m}$ dimensional control and a minimum surface roughness as low as $2 \mu \mathrm{m}$. However, more aggressive roughness $(<1 \mu \mathrm{m})$ are today only achievable using SLA technology. It explains why the best 3D printed waveguides working $>200 \mathrm{GHz}$ reported in the literature have been achieved using metal coated dielectric part printed using SLA [9].

In order to achieve better surface roughness, one solution could consist in reducing the size of the powder particles used by SLS or SLM technologies. This is exactly what MLS technology is offering. MLS has been recently introduced by 3D Microprint [13] and is today the most accurate technology to $3 \mathrm{D}$ print solid metal structures. The main difference of MLS compared to SLS is the use of stainless steel powder material which exhibit particle size smaller than $5 \mu \mathrm{m}$ (in comparison with $\sim 20 \mu \mathrm{m}$ for SLS). Consequently, the 3D part is built with $5 \mu \mathrm{m}$ thick layer leading to improved dimensional accuracy and surface roughness.

Promising results have already been reported in the literature up to $100 \mathrm{GHz}$ using MLS technology to achieve the highest frequency metal 3D printed filter so far reported [14]. We propose to assess in the next section achievable performances up to $325 \mathrm{GHz}$ using waveguide and horn antenna test vehicles.

\section{Evaluation of Micro Laser Sintering Metal 3D- PRINTING TECHNOLOGY UP TO $325 \mathrm{GHz}$}

\section{A. WR5 Waveguide Test Vehicle}

In order to asses MLS technology, a WR5 back-to-back modules has been designed, simulated and characterized in Gband (140-220 GHz). Since the thickness of 3D printed metallic part is limited, we used $\mathrm{H}$ plan and $\mathrm{E}$ plan $90^{\circ}$ bend in order to be able to integrate long enough straight waveguide $(7.2 \mathrm{~mm}$ in our case) in the module thickness. Two WR5 waveguide flanges are then integrated in the module in order to connect the measurement system. The figure below gives detailed dimensions of waveguide cavities curved in metallic blocks:

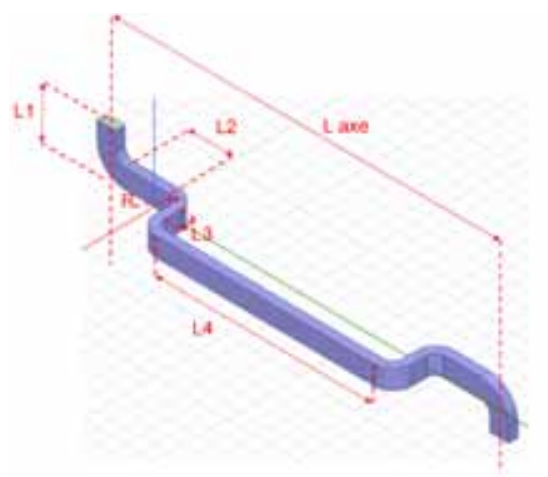

Fig. 1. Dimensional description of WR5 waveguides

Dimensions are detailed below:

Table 2. Optimized dimensions of the bended WR5 waveguide

\begin{tabular}{|c|c|}
\hline L1 $(\mathrm{mm})$ & 3.162 \\
\hline L2 $(\mathrm{mm})$ & 3.12 \\
\hline L3 $(\mathrm{mm})$ & 1 \\
\hline L4 $(\mathrm{mm})$ & 7.2 \\
\hline L axe $(\mathrm{mm})$ & 19.275 \\
\hline R $(\mathrm{mm})$ & 1.296 \\
\hline
\end{tabular}

Back-to-back S-parameters measurement were performed in G-band, using a set of Olesson 140-220 GHz extension heads driven by a Rohde \& Schwarz VNA. Thru Reflect Match (TRM) calibration was used prior to the measurements.

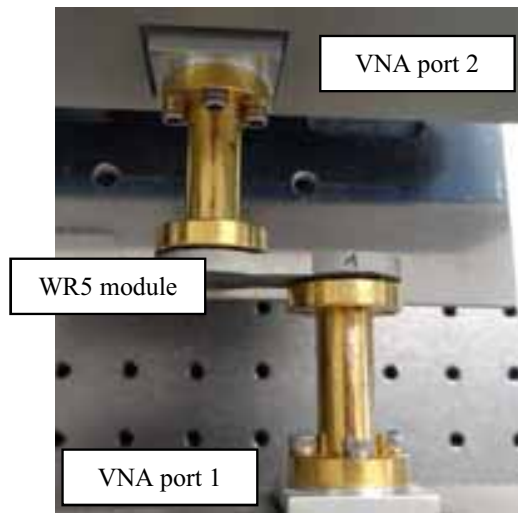

Fig. 2. Picture of the back-to-back WR5 module measurement 
Measurements demonstrate promising performances, validating the suitability of MLS technology at G-band. As illustrated in Fig. 3, measured matching is $<-13 \mathrm{~dB}$ in G-band and an acceptable agreement is obtained with simulated data from Ansys HFSS.

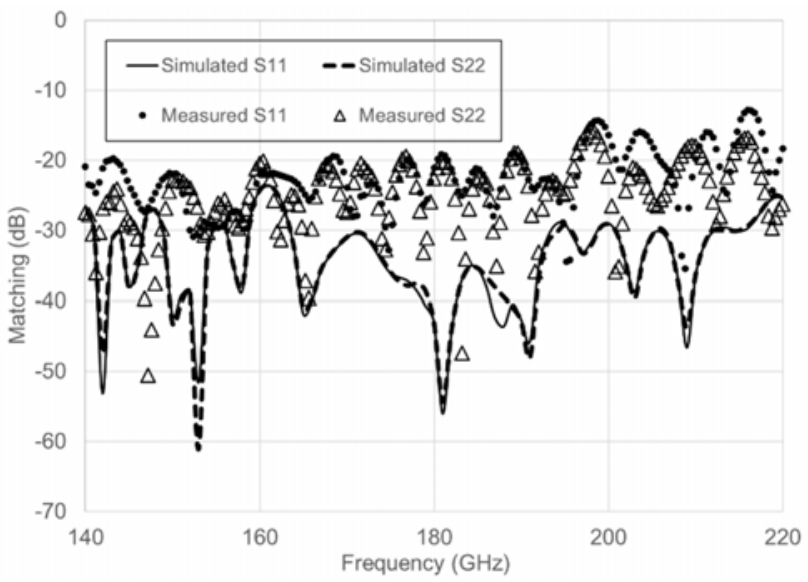

Fig. 3. Measured and simulated matching of WR5 waveguide test vehicle

A fair agreement has also been achieved up to $220 \mathrm{GHz}$ between measured and simulated insertion loss as illustrated in Fig. 4.

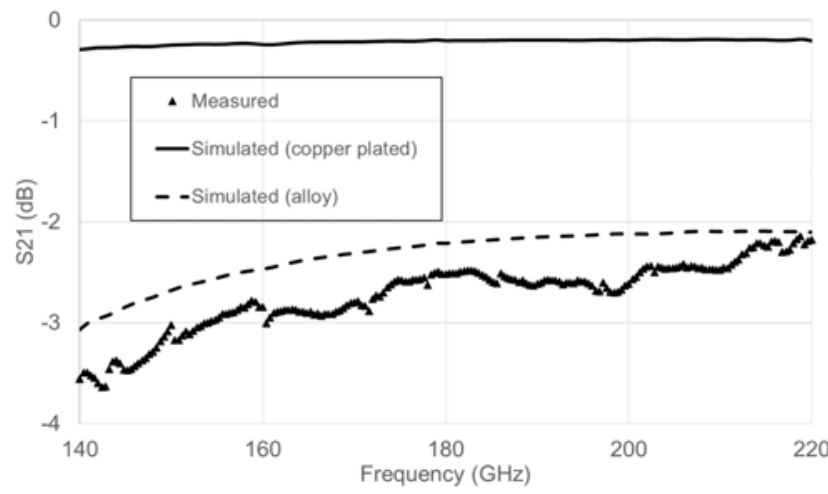

Fig. 4. Measured and simulated insertion loss of WR5 waveguide test vehicle

A discrepancy below $0.5 \mathrm{~dB}$ is observed between simulated and measured insertion losses up to $220 \mathrm{GHz}$. These results confirm the excellent accuracy and surface roughness that can be expected from MLS technology. It clearly demonstrates the suitability of MLS technology to operate beyond D-band in order to extend the capability of metallic 3D-Printing technology.

However, even if those results are impressive we have to underline here that the achieved insertion loss $(90 \mathrm{~dB} / \mathrm{m})$ does not compete with commercial WR5 waveguide $(20 \mathrm{~dB} / \mathrm{m})$ manufactured using standard processing. This limitation is related to the 17-4PH stainless steel powder material used by MLS. The type of stainless steel is a chromium nickel copper alloyed stainless steel, containing $17 \%$ chromium, $4 \%$ nickel, $4 \%$ copper and $0.3 \%$ niobium. This alloy exhibits a limited electrical conductivity of $1.25 \times 10 \mathrm{e} 7 \mathrm{~S} / \mathrm{m}$ which explains the experienced larger insertion loss. As proposed in [14], this issue could be easily solved by electroplating a few $\mu \mathrm{m}$ thick copper layer on the surface of the 3D printed part (increasing the electrical conductivity by a factor of $\sim 5$ ). As illustrated Fig. 4 by simulation, insertion loss at $220 \mathrm{GHz}$ could then be reduced from $\sim 2.5 \mathrm{~dB}$ down to $\sim 0.3 \mathrm{~dB}$ (competing with commercial waveguide manufacturing technology).

\section{B. $240 \mathrm{GHz}$ Horn Antenna Test Vehicle}

In order to assess achievable performances of MLS technology at even higher frequency, we leveraged an existing $240 \mathrm{GHz}$ choke horn antenna design [15]. This antenna was designed to have a $12 \mathrm{dBi}$ gain over the $200 \mathrm{GHz}$ to $280 \mathrm{GHz}$ bandwidth, with $\mathrm{w}=0.3 \mathrm{~mm}$, horn apperture $\mathrm{A}=4.486 \mathrm{~mm}$, horn length $\mathrm{L}=9.853 \mathrm{~mm}$. A caption of the $3 \mathrm{D}$ simulated half-model is shown in Fig. 5, where the cross-section is also presented.

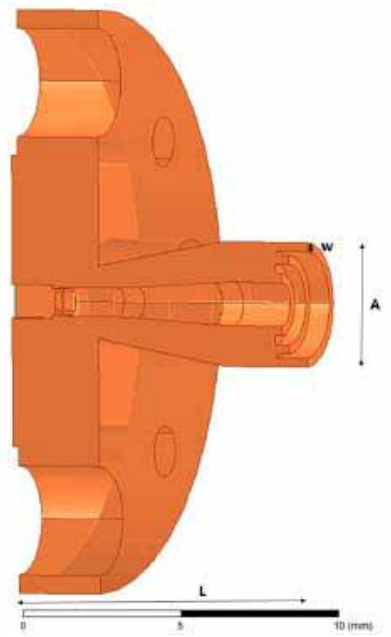

Fig. 5. 3D-(Half) Model of the $240 \mathrm{GHz}$ horn antenna [15]

This antenna design has been manufactured using metal coated SLA [15], SLM [16] and during this work MLS technology. Manufactured samples are presented Fig. 6. It is impressive to observe how MLS technology (without post processing) enables to drastically improve achievable surface roughness of SLM parts (competing with metal coated SLA technology).

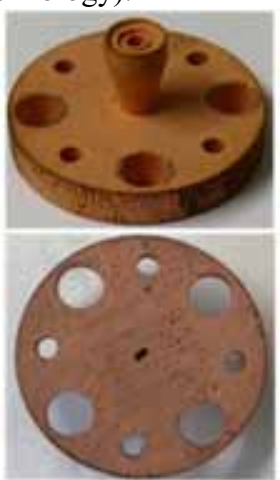

(a)

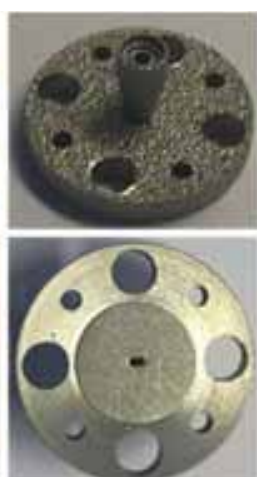

(b)

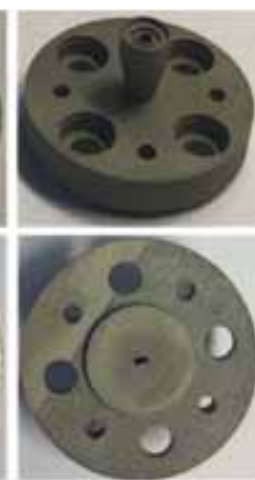

(c)
Fig. 6. Prototyped horn antennas using SLA (a), SLM (b) and MLS (c) 3DPrinting technologies 
Those three antenna prototypes have been characterized with the in-house set up presented in [16]. Promising performances have been achieved by the MLS sample. We present Fig. 8 the realized gain achieved in $\mathrm{H}$ plan at $320 \mathrm{GHz}$ for MLS antenna samples and the simulation results (achieved using Ansys HFSS). Excellent agreement is obtained between measurement and simulation confirming the excellent surface roughness and accuracy that can be expected from MLS technology.

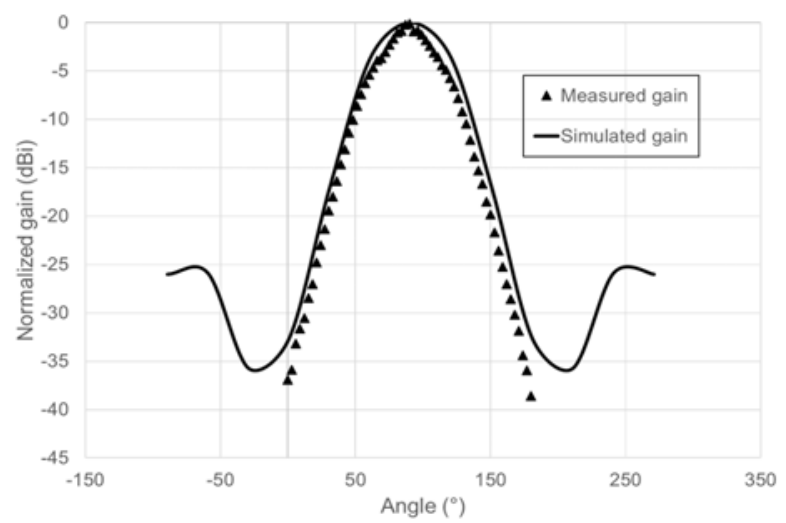

Fig. 7. Measured horn antennas realized gain in $\mathrm{H}$ plan at $300 \mathrm{GHz}$ for MLS 3D-Printing technologies

We present Fig. 8 the broadside realized gain in the $220-$ $325 \mathrm{GHz}$ band for the SLA, SLM, MLS samples and the initial simulation performed using Ansys HFSS. SLM sample demonstrated poor performances that can be easily explained by achieved surface roughness, SLA sample exhibits the best performances (agreeing very well with simulation results) and MLS sample competes with SLA ones achieving a realized gain only $\sim 1 \mathrm{~dB}$ lower.

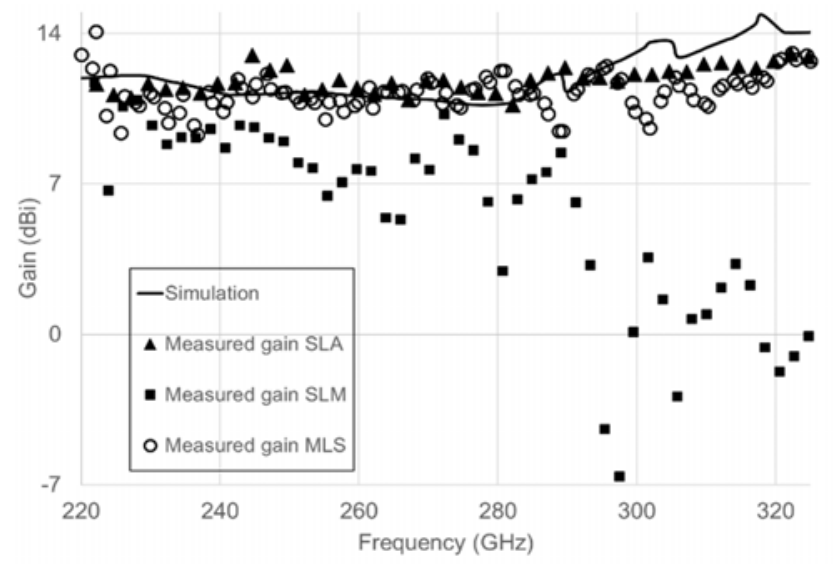

Fig. 8. Simulated broadside horn antenna realized gain and measurements for SLA, SLM and MLS 3D-Printing technologies

This benchmark demonstrates for the first time the possibility for a metallic 3D-Printing technology to compete with metal coated SLA technology. It confirms once again the excellent accuracy and surface roughness that can be expected from MLS technology.

\section{CONCLUSION}

In this paper, we reported for the first time the assessment of innovative MLS 3D-Printing technology up to $320 \mathrm{GHz}$. Promising performances have been reported, clearly confirming the excellent accuracy and surface roughness $(<1$ $\mu \mathrm{m}$ ) that can be expected from MLS technology (without any post processing). It demonstrates for the first time the capacity of a metal 3D-Printing technology to compete with metal coated SLA one. However, achieved waveguide losses are today limited by the properties of the stainless steel powder material imposed by the MLS process. Consequently, as suggested in [14] it would be interesting to add copper electroplating post processing to MLS manufacturing in order to improve further achievable performances up to $320 \mathrm{GHz}$.

\section{REFERENCES}

[1] IEEE802.15 documents. Task Group 3d 100 Gbit/s Wireless (TG3d (100G)). http://www.ieee802.org/15/pub/index TG3d.html

[2] P. Chevalier et al., "SiGe BiCMOS Current Status and Future Trends in Europe," 2018 IEEE BiCMOS Compd. Semicond. Integr. Circuits Technol. Symp. BCICTS 2018, pp. 64-71, 2018.

[3] A. Jebril, M. Lucente, T. Rossi, M. Ruggieri, and L. Zuliani, "AeroWAVE: A W-band preliminary test using HAP," IEEE Aerosp. Conf. Proc., vol. 2006, p. 6, 2006.

[4] B. Zhang et al., "Integration of a $140 \mathrm{GHz}$ Packaged LTCC Grid Array Antenna With an InP Detector," in IEEE Transactions on Components, Packaging and Manufacturing Technology, vol. 5, no. 8, pp. 1060-1068, Aug. 2015.

[5] S. E. Gunnarsson, N. Wadefalk, I. Angelov, H. Zirath, I. Kallfass and A. Leuther, "A $220 \mathrm{GHz}$ (G-Band) Microstrip MMIC Single-Ended Resistive Mixer," in IEEE Microwave and Wireless Components Letters, vol. 18, no. 3, pp. 215-217, March 2008.

[6] P. Sobis, Advanced Schottky Diode Receiver Front-Ends for Terahertz Applications, Thesis Report, May 2011.

[7] M. Alonso-del Pino, C. Jung-Kubiak, T. Reck, C. Lee and G Chattopadhyay, "Micromachining for Advanced Terahertz: Interconnects and Packaging Techniques at Terahertz Frequencies," in IEEE Microwave Magazine, vol. 21, no. 1, pp. 18-34, Jan. 2020.

[8] B. Zhang, Y. Guo, H. Zirath and Y. P. Zhang, "Investigation on 3-DPrinting Technologies for Millimeter- Wave and Terahertz Applications," in Proceedings of the IEEE, vol. 105, no. 4, pp. 723-736, April 2017.

[9] A. von Bieren et al., "Monolithic metal-coated plastic components for mm-Wave applications," in Proc. 39th Int. Conf. Infr. Millim. Terahertz Waves, Tucson, TX, USA, Sep. 2014, pp. 1-2.

[10] B. Zhang et al., "Metallic 3D printed antennas for millimeter- and submillimeterwave applications," IEEE Trans. THz Sci. Technol., vol. 6, no. 4, pp. 592-600, Jul. 2016

[11] Micro Machined Process. BINC, accessed on Dec. 15, 2015. [Online]. Available: http://www.binc.biz/gb/index.php

[12] B. Zhang and H. Zirath, "Metallic 3-D printed rectangular waveguides for millimeter-wave applications," IEEE Comp. Packag. Manuf. Technol., vol. 6, no. 5, pp. 796-804, May 2016.

[13] The 3D Microprint $\mathrm{GmbH}$ website. [Online]. Available: https://www.3dmicroprint.com/

[14] M. Salek et al., "W-Band Waveguide Bandpass Filters Fabricated by Micro Laser Sintering," in IEEE Transactions on Circuits and Systems II: Express Briefs, vol. 66, no. 1, pp. 61-65, Jan. 2019.

[15] C. Biurrun-Quel et al., "Characterization of 3D-Printed Choke Horn Antenna for 5G Backhaul Applications", $13^{\text {th }}$ European Conference on Antennas and Propagation (EuCAP), Krakow, April 2019.

[16] C. B. Goncalves, E. Lacombe, C. del Rio, F. Gianesello, C. Luxey and G. Ducournau, "Compact Antennas Pattern Measurement Setup at 240 GHz," 2018 43rd International Conference on Infrared, Millimeter, and Terahertz Waves (IRMMW-THz), Nagoya, 2018, pp. 1-2. 